\title{
VIRTUAL COORDINATE MEASURING MACHINE BUILT USING LASERTRACER SYSTEM AND SPHERICAL STANDARD
}

\author{
Jerzy Sładek, Adam Gąska, Magdalena Olszewska, Robert Kupiec, Marcin Krawczyk \\ Cracow University of Technology, Faculty of Mechanical Engineering, Aleja Jana Pawła II 37, 31 -864 Kraków, Poland \\ (هsladek@mech.pk.edu.pl, +48 12374 3230,agaska@mech.pk.edu.pl,molszewska@mech.pk.edu.pl, rkupiec@mech.pk.edu.pl, \\ mkrawczyk@mech.pk.edu.pl)
}

\begin{abstract}
Improvements of modern manufacturing techniques implies more efficient production but also new challenges for coordinate metrologists. The crucial task here is a coordinate measurement accuracy assessment. It is important because according to technological requirements, measurements are useful only when they are stated with their accuracy. Currently used methods for the measurements accuracy estimation are difficult to implement and time consuming. It is therefore important to implement correct and validated methods that will also be easy to implement. The method presented in this paper is one of them. It is an on-line accuracy estimation method based on the virtual CMM idea. A model is built using a modern LaserTracer system and a common test sphere and its implementation lasts less than one day. Results obtained using the presented method are comparable to results of commonly used uncertainty estimation methods which proves its correct functioning. Its properties predispose it to be widely used both in laboratory and industrial conditions.
\end{abstract}

Keywords: virtual CMM model, Monte Carlo method, LaserTracer.

(C) 2013 Polish Academy of Sciences. All rights reserved

\section{Introduction}

Assessment of dimensional compliance with geometrical product specification (GPS) is becoming a crucial task for production engineering. The risk of wrong decisions carries consequences that are observed as a wave of product complaints in main branches of industry. The importance here is given to the possibility of determining the accuracy of measurement, because according to technological requirements, results of measurements are useful only when they are given with their accuracy. In the case of coordinate measuring techniques (CMT) it is a task particularly difficult and not always straightforward [1-3]. Therefore, users of this technique, as well as manufacturers of these measuring systems often overlook the problem of measurement accuracy giving in exchange the accuracy of measuring devices. This accuracy is determined then for the realization of the selected task as a measurement of distance and is given as the maximum permissible error (MPE). Thus defined, it is significantly different from the accuracy of the real measuring task, and may lead to bad decisions in the determination of compliance with the specifications of the product [2, 4, 5-9]. Moreover, when the MPE is used instead of real task-specific accuracy, all measuring tasks are simplified to measurement of distance, which is not consistent with the coordinate measuring technique nature, which is the measurement of values of coordinates of the measuring point.

It is therefore essential, to implement new, correct and metrologically validated methods. Those currently used are difficult, require knowledge and experience in the field of measurement and are hugely time consuming [10, 11]. Considering the level of metrological expertise, their implementation is most advanced in the German industry, which is connected 
with the studies issued by VDI [12]. Analyzing the problem of assessing the accuracy of coordinate measurements, it can be divided into two main methods, the substitution method and the multiple measurement method - the best described and documented by normative establishments [10]. Descriptions of their use can be found in [13-15]. Another group consists of analytical methods based on calculation of uncertainty of indirect measurement using the geometrical relations between them, performed similarly to the classical estimation of uncertainty of measurement done i.e. by a micrometer. These methods are based on studies of Pressel [16], Hernla [17, 18] and Jakubiec [19, 20]. However, these methods are difficult to use in practice and they present an approach similar to methods based on expert knowledge [10].

Nowadays, a new trend in accuracy estimation can be seen and it is connected with creation of simulative methods. In practice, these methods require the development of socalled virtual measuring machines used to assess the on-line accuracy of measurement. These methods are so far the most accurate as they are based on reproducibility of the measuring point idea and because of it, they are consistent with the coordinate measuring technique nature. Two main concepts could be pointed out here: identification of sources of CMM errors (including kinematic errors) and errors of the probe head (the PTB Novel method can be taken as an example of this method [21,22]) and the second one - the so called Matrix Method (MM), created at Cracow University of Technology and based on identification of errors at selected reference points in machine measuring area and construction of a CMM model using different interpolation methods (for example artificial neural networks [23]). Both concepts are continually developed. They are used for example to create virtual models of Coordinate Measuring Arms [24]; MM is also implemented on large scale machines [25, 26], here, a possibility of use of LaserTracer (LT) and LaserTracer for the construction of reference points grid was noticed. The experiments with using laser tracking systems and the numerical Monte Carlo method (MCM) has lead to development of a new accuracy estimation system. It can be used both in laboratory and industrial conditions. The main assumptions of the created model, its validation in laboratory conditions and directions of its further development are the main topics of this paper.

\section{Main assumptions of the created virtual CMM model}

Nowadays, in typical measuring machines the systematic errors are compensated for the kinematic system (CAA correction matrix), probe head (dynamic error correction matrix) and for components originating from the environmental influences exemplified by the temperature correction (e.g. ACTIV). Therefore, the authors decided to transfer the problem of modeling of accuracy of the CMM to the field of residual errors that remain uncompensated.

Given the above facts, it is assumed that the presented simulation model is developed for modern metrological systems, equipped with a full, active error compensation (so it is designed for about $90 \%$ of the total number of CMMs). The described model of a CMM consists of two basic modules:

- a module that simulates the residual and random errors dependent on the kinematic system of the CMM;

- a module that simulates the work of the probe head.

The first module of the CMM model is built by describing each point on the grid of reference points by the probability distribution ( $t$ distribution in case of this model) with which it is reproduced on a machine. But it is necessary to have a system that would allow experimental determination of distribution of errors in reference points, with the required accuracy. Currently, only the LaserTracer (LT) meets the requirements concerning 
the accuracy that allows to do so. This is why it was used (combined with the multilateration technique) for experimental determination of distribution of errors in reference points (Fig. 1).

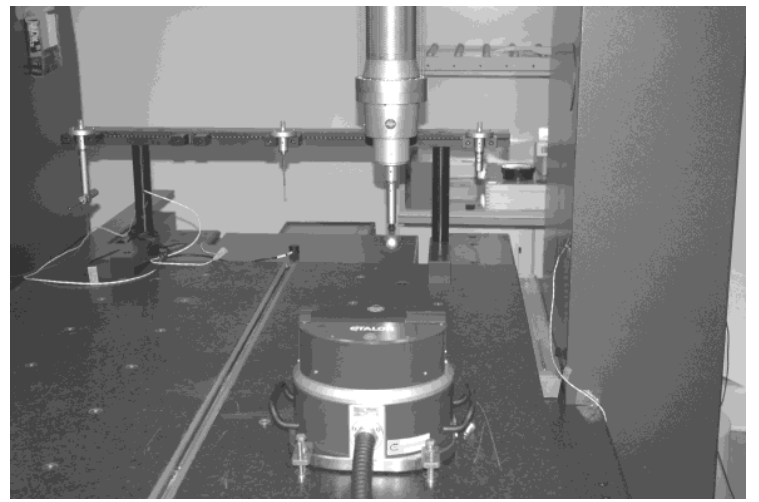

Fig. 1. Cooperation between the CMM and LaserTracer.

The LaserTracer (Fig. 2) is an interferometric device which allows tracking of movements of a reflector mounted on the probe head of the measuring machine or on the tool holder of the machining tool. The accuracy of this device is given by:

$$
U=0.2+0.3 * L / 1000 \mu \mathrm{m},
$$

where $L$ is the measured length given in $\mathrm{mm}$.

Such high accuracy can be achieved thanks to a novel solution in its construction based on a reference sphere whose form errors do not exceed $30 \mathrm{~nm}$. This sphere lies in the center of rotation of the LT rotary system.

The experiment aiming at determination of the mentioned reference grid consisted of repeated approach to the considered points from different directions. In place of the stylus the "cat eye" retro reflector was mounted. The position of the retro reflector was tracked in the dynamic mode by the LaserTracer installed in the measuring volume of the machine. After a sequence of approaches at the point, the machine reached the next one and the cycle was repeated. The whole measurement sequence was repeated five times, each in a different position of LT, in order to determine the coordinates of points using the multilateration method [27].

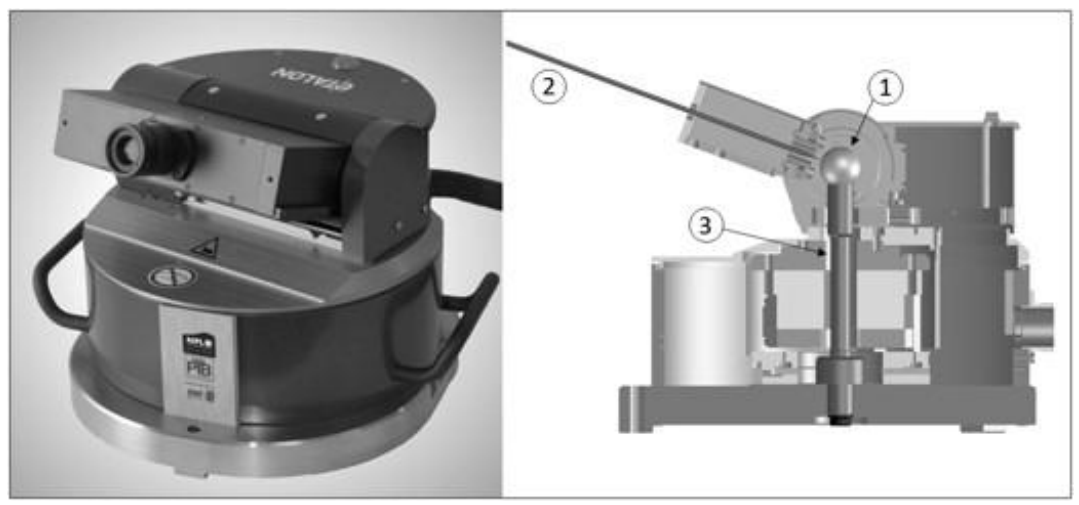

Fig. 2. LaserTracer: 1) reference sphere, 2) laser beam, 3) LT column [28].

Multilateration is a measuring method that determines either two or three dimensional coordinates by combining only length measurements made from fixed points. It is a method which is broadly used in the Global Positioning System (GPS). The measuring system 
determines the coordinates of the targets and tracking stations by using a non-linear least squares algorithm.

The least squares algorithm is used here to fit all the measurement variables to all the system variables. The measurement variables are defined as all the distances $\left(l_{i j}\right)$ from tracer zero points to all the targets and the system variables are the four laser tracer position coordinates $\left(X_{i}, Y_{i}, Z_{i}\right)$, the "dead path" length of each tracer $\left(L_{i}\right)$ and the $x, y, z$ coordinates of target $\left(x_{j}, y_{j}, z_{j}\right)$. This is done by minimizing the residuals of the function given in (2). The residual $e_{i j}$, for the $i$-th measurement station and $j$-th target point, is [29]:

$$
e_{i j}=\sqrt{\left(x_{j}-X_{i}\right)^{2}+\left(y_{j}-Y_{i}\right)^{2}+\left(z_{j}-Z_{i}\right)^{2}}-\left(l_{i j}-L_{i}\right) .
$$

The second module forming part of the described measuring virtual machine is a module responsible for the simulation of probe head of CMM. To describe this system the Probe Error Function (PEF) described in [23] has been used. The module gets the values of the individual errors of the PEF through Monte Carlo simulations.

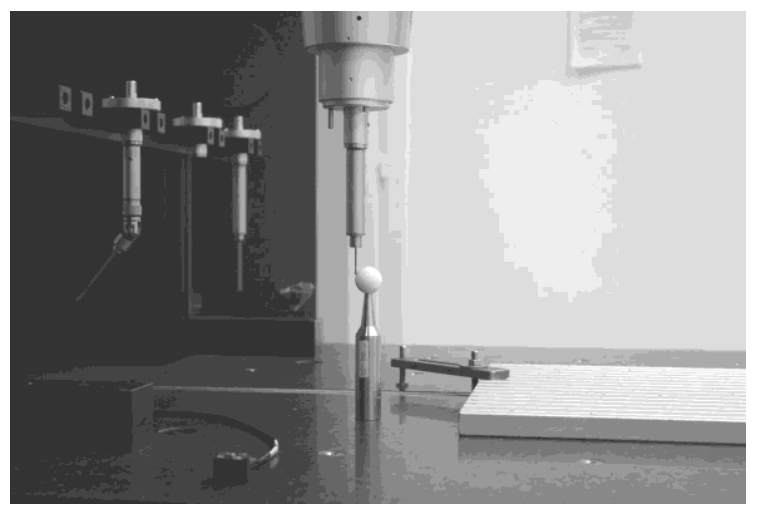

Fig. 3. Measurements of test sphere performed on Leitz PMM 12106 machine equipped with a Leitz measuring probe head.

Data used to build this module was collected by multiple measurement of the test sphere (Fig. 3). The standard was measured each time in 163 points that create the reference grid for values of PEF. The reference grid is schematically shown in Fig. 4. Proper operation of the model is provided by using a test sphere with suitably small form errors (its diameter should be smaller than $30 \mathrm{~mm}$ ).

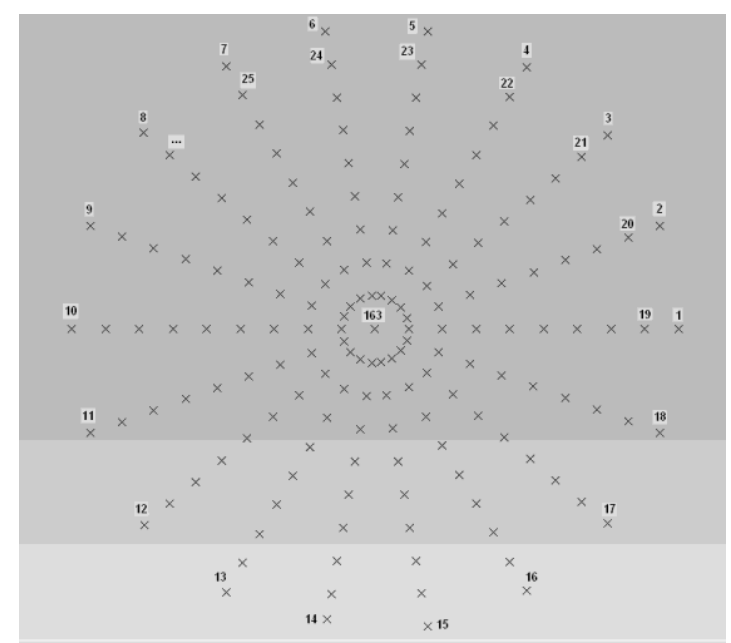

Fig. 4. Measuring sequence, points indicated by " $x$ " are nods of reference grid (view from top of the test sphere). 
The problem of interpolating values of errors between nodes of the reference grid arises for both described modules. It is obvious that in real measurements the majority of measuring points would lie between nodes of the reference grid. In order to get the variability of errors in these points, the authors used different interpolation methods. In case of kinematic system errors the b-spline and "nearest-neighbor" interpolation methods were used, while in case of probe head error interpolation, the bilinear interpolation was used.

\section{Results of error identification}

All tests were performed at the Laboratory of Coordinate Metrology at Cracow University of Technology, which is a laboratory accredited according to the ISO 17025 standard. The modeled machine was Leitz PMM 12106 equipped with a Leitz scanning probe head. Below, exemplary results of kinematic residual errors and probe head error identification were presented. For more detailed results see [27, 30]. Results presented in Table 1 were obtained using 52 reference points distributed uniformly across the modeled measuring volume of the machine. At each point, a sequence of 14 approaches from different directions was performed.

Table 1. Results of kinematic residual errors identification for chosen reference points: $X, Y, Z$ - nominal coordinates of reference points, $\mathrm{mm} ; d(\mathrm{X}), d(\mathrm{Y}), d(\mathrm{Z})$ - standard deviations of reproduction of certain coordinate of points, $\mathrm{mm}$.

\begin{tabular}{|c|c|c|c|c|c|c|c|c|c|c|c|c|c|}
\hline No. & $\mathrm{X}$ & $\mathrm{Y}$ & $\mathrm{Z}$ & $d(\mathrm{X})$ & $d(\mathrm{Y})$ & $d(\mathrm{Z})$ & No. & $\mathrm{X}$ & $\mathrm{Y}$ & $\mathrm{Z}$ & $d(\mathrm{X})$ & $d(\mathrm{Y})$ & $d(\mathrm{Z})$ \\
\hline 21 & 475 & 190 & 200 & 0.00055 & 0.00060 & 0.00044 & 31 & 150 & 50 & 350 & 0.00069 & 0.00039 & 0.00147 \\
22 & 475 & 325 & 200 & 0.00044 & 0.00052 & 0.00079 & 32 & 150 & 325 & 350 & 0.00021 & 0.00037 & 0.00086 \\
23 & 475 & 460 & 200 & 0.00037 & 0.00061 & 0.00038 & 33 & 150 & 600 & 350 & 0.00018 & 0.00054 & 0.00056 \\
24 & 475 & 600 & 200 & 0.00031 & 0.00036 & 0.00104 & 34 & 310 & 460 & 350 & 0.00028 & 0.00030 & 0.00039 \\
25 & 640 & 460 & 200 & 0.00079 & 0.00084 & 0.00037 & 35 & 310 & 190 & 350 & 0.00023 & 0.00053 & 0.00127 \\
26 & 640 & 325 & 200 & 0.00094 & 0.00071 & 0.00075 & 36 & 475 & 50 & 350 & 0.00118 & 0.00070 & 0.00061 \\
27 & 640 & 190 & 200 & 0.00107 & 0.00071 & 0.00042 & 37 & 475 & 325 & 350 & 0.00046 & 0.00031 & 0.00096 \\
28 & 800 & 50 & 200 & 0.00185 & 0.00137 & 0.00054 & 38 & 475 & 600 & 350 & 0.00034 & 0.00039 & 0.00049 \\
29 & 800 & 325 & 200 & 0.00248 & 0.00136 & 0.00057 & 39 & 640 & 460 & 350 & 0.00096 & 0.00076 & 0.00058 \\
30 & 800 & 600 & 200 & 0.00175 & 0.00175 & 0.00100 & 40 & 640 & 190 & 350 & 0.00109 & 0.00078 & 0.00058 \\
\hline
\end{tabular}

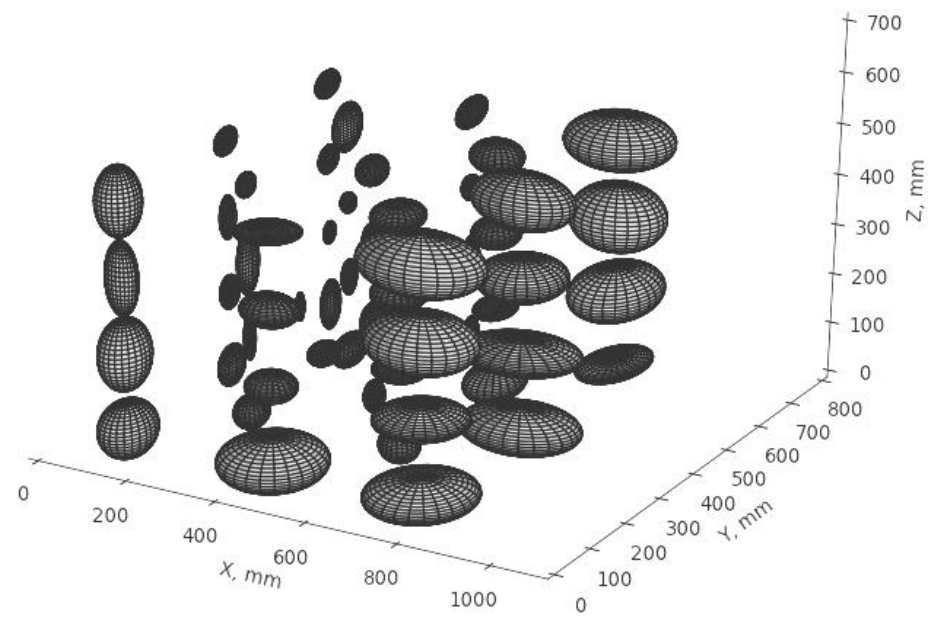

Fig. 5. Values of residual errors presented as ellipsoids of the point reproduction error. 
In order to create a model of error of the probe head, an appropriate set of data showing a general picture of probe head errors depending on the angles $\alpha$ and $\beta$ where gathered with the use of a test sphere of diameter $d=24.9944 \mathrm{~mm}$. In this experiment, the overall number of 163 points equally distributed around the test sphere was measured 15 times. Table 2 and Fig. 6 present the results.

Table 2. Results of measurements of a test sphere for chosen angles of deflection of stylus $\alpha$ and $\beta$. Mean radial error and standard deviation stated in mm.

\begin{tabular}{|c|c|c|c|c|c|c|c|}
\hline$\alpha$ & $\beta$ & Mean error & Standard dev & $\alpha$ & $\beta$ & Mean error & Standard dev \\
\hline 0 & 10 & 0.00004 & 0.00005 & 180 & 10 & 0.00016 & 0.00002 \\
20 & 10 & -0.00022 & 0.00004 & 200 & 10 & -0.00001 & 0.00004 \\
40 & 10 & -0.00043 & 0.00004 & 220 & 10 & -0.00025 & 0.00004 \\
60 & 10 & -0.00021 & 0.00005 & 240 & 10 & -0.00014 & 0.00004 \\
80 & 10 & 0.00009 & 0.00004 & 260 & 10 & -0.00012 & 0.00005 \\
100 & 10 & 0.00021 & 0.00004 & 280 & 10 & -0.00015 & 0.00003 \\
120 & 10 & 0.00017 & 0.00004 & 300 & 10 & -0.00002 & 0.00003 \\
140 & 10 & 0.00021 & 0.00005 & 320 & 10 & 0.00020 & 0.00002 \\
160 & 10 & 0.00008 & 0.00004 & 340 & 10 & 0.00031 & 0.00003 \\
\hline
\end{tabular}

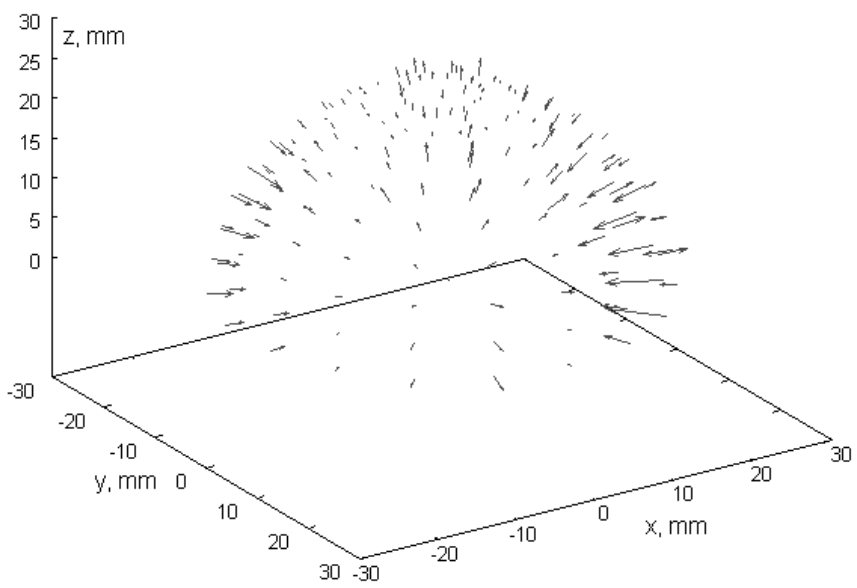

Fig. 6. Probe head errors depending on angles of deflection of stylus $\alpha, \beta$.

\section{Verification of the created model}

Verification of the model consisted of two stages. In the first one, the developed model was tested according to VDI/VDE guidelines [12]. The second stage consisted of performing common metrological tasks such as measurements of the point-to-point distance, the plane-toplane distance, the diameter of the sphere, the form error of the sphere and the distance between the centres of two spheres. Then, for each measurement the standard uncertainty was determined according to the methodology used in the classical methods of determining the accuracy of measurements. The uncertainty determined in the classical way was compared with the uncertainty obtained by the developed simulation model.

All of performed tests proved correct functioning of the presented model. As an example, the results of measurements of diameter and form errors of standard cylinder performed according to [12], with various number of measuring points measured on the surface of cylinder (Fig. 7) were presented in Table 3. 


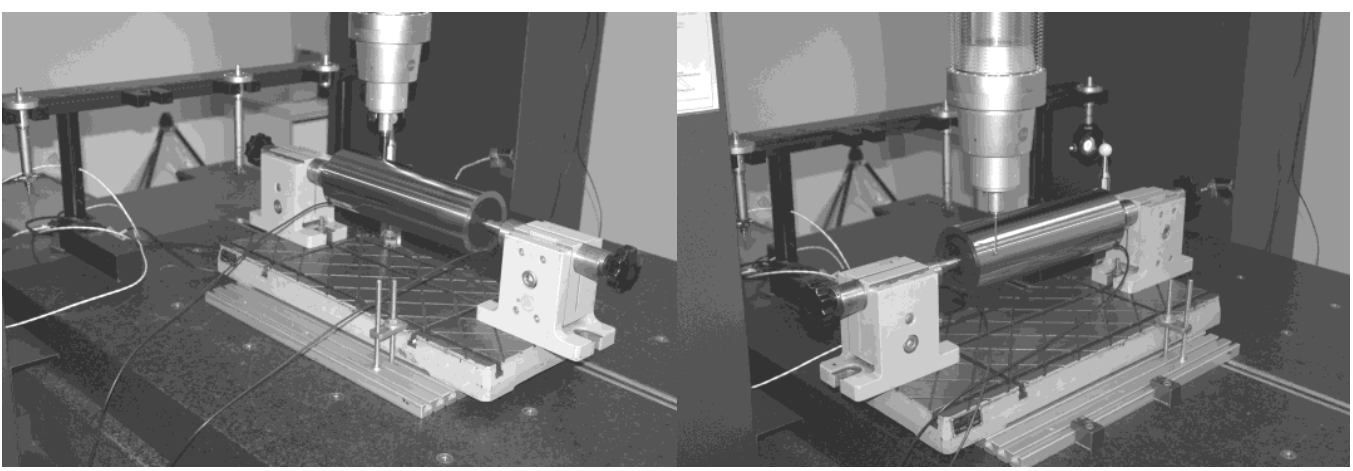

Fig. 7. Measurements of a standard cylinder.

Table 3. Results of measurements of a standard cylinder. Symbols in Table: $y$ - the measured value, $y_{k}$ - the value obtained in the calibration process of standard, $U$-expanded uncertainty of measurement (determined using the simulation model that is being checked), $U_{k}$ - expanded uncertainty of the calibration of standard.

\begin{tabular}{|c|c|c|c|c|c|}
\hline No. of measuring points & Measured feature & $y, \mathrm{~mm}$ & $y_{k}, \mathrm{~mm}$ & $U, \mathrm{~mm}$ & $U_{k}, \mathrm{~mm}$ \\
\hline \multirow{2}{*}{9} & Diameter & 100,2460 & 100,2455 & 0,0009 & 0,0007 \\
\cline { 2 - 6 } & Form error & 0,0015 & 0,0016 & 0,0006 & 0,0003 \\
\hline \multirow{2}{*}{15} & Diameter & 100,2460 & 100,2455 & 0,0008 & 0,0007 \\
\cline { 2 - 6 } & Form error & 0,0017 & 0,0016 & 0,0005 & 0,0003 \\
\hline \multirow{2}{*}{24} & Diameter & 100,2462 & 100,2455 & 0,0007 & 0,0007 \\
\cline { 2 - 6 } & Form error & 0,0016 & 0,0016 & 0,0005 & 0,0003 \\
\hline
\end{tabular}

All of the presented results of measurements satisfy the formula (3), which means that the simulation model is considered to be operating correctly according to [12]:

$$
\left|y_{k}-y\right| \leq U_{k}+U \text {. }
$$

\section{Directions of further development of the model}

The main task for the Virtual MCM PK model that needs realization in the near future is its implementation in an industrial environment.

All industrial plants using coordinate systems should be treated as potential recipients of the presented model. The issue of reporting of the measurement results with the corresponding uncertainty, as already demonstrated in the first chapter, is a fundamental issue in the field of metrology and widely understood quality control. This case, until now, due to the complex methodology of determination of uncertainty and its huge time-consuming rate, is underestimated in most companies. The prevalence of the described method can help to improve this situation, and thus minimize the amount of erroneous assessments of compliance of the products with their geometrical specification.

It should be also noticed that there is a possibility of system implementation in a single day. It is the only model of a virtual measuring machine which can be implemented in such a short time. This feature also predisposes it to be used on a large scale in the industry.

Another possible direction of development is the application of the created model combined with an I++ Simulator (software used for simulation of various types of CMMs) to determine the optimal measurement strategy. With a model of the kinematic system's residual errors and the probe head errors it would be possible to determine the optimum number of points and their distribution on the measured object. Knowing the distribution of errors in the machine measuring space, it could be also possible to locate the measured object in the place in which it should be mounted in order to maximize the measurement accuracy. 
Research on reduction of the number of data needed to create the model could also be undertaken. As it was shown in the tests carried out by the authors in [31], a reduction of points on the test sphere is possible. According to the authors it should also be possible to reduce the number of reference points on which the variability of measuring point reproducibility was described. What should be investigated is whether and how the reduction of data points affects the proper functioning of the described model, and then, depending on the required precision of estimation of measurement uncertainty, the number of points that can provide it should be chosen.

\section{Conclusion}

Basing on the considerations and results of experiments presented in this paper it has to be noticed that it is possible to develop a comprehensive method of assessing the CMM accuracy based on the determination of residual errors at the reference points.

For the modeled measuring machine, the hypothesis about possibility of transferring the problem of modeling of accuracy of measuring machines to the area of random errors turned out to be correct, assuming correct functioning of the compensation systems of systematic errors of the machine.

The model presented in this work is less laborious than models based on the modeling of individual components of the systematic errors of the measuring equipment. Thanks to this, it can be easily implemented in industrial or laboratory use.

The data necessary to build the described model, thanks to the LaserTracer and combined with an effective working plan can be collected even in a single day. Such rapid process of a model creation for a certain machine can contribute to the increase of popularity of the developed method, which models measurements in the manner most similar to the nature of coordinate measurements.

A virtual model of the CMM based on the modeling of residual errors with the use of the LaserTracer system and multilateration methods allows the construction of an on-line virtual machine, which combined with a typical metrological software enables the determination of the uncertainty of modeled measurement in a quasi-real time. This method is consistent with the essence of the coordinate measuring technique which is a measurement of a single point. This is why it is the most effective method among the known methods of uncertainty estimation.

\section{References}

[1] Barini, E.M., Tosello, G., De Chiffre, L. (2010). Uncertainty analysis of point-by-point sampling complex surfaces using touch probe CMMs: DOE for complex surfaces verification with CMM. Precis. Eng., 34(1), 16-21.

[2] Beaman, J., Morse, E. (2010). Experimental evaluation of software estimates of task specific measurement uncertainty for CMMs. Precis. Eng., 34(1), 28-33.

[3] Bleys, P., Van Gestel, N., Cuypers, S., Kruth, J.P. (2007). Uncertainty due to Limited Sampling of Circular Features on Coordinate Measuring Machine. In Proc. of the $10^{\text {th }}$ CIRP Conference on Computer Aided Tolerancing, Erlangen, Germany.

[4] Germani, M., Mandorli, F., Mengoni, M., Raffaeli, R. (2010). CAD-based environment to bridge the gap between product design and tolerance control. Precis. Eng., 34(1), 7-15.

[5] Pedone, P., Romano, D. (2011). Designing small samples for form error estimation with coordinate measuring machines. Precis. Eng., 35(2), 262-270. 
[6] Phillips, S.D., Borchardt, B., Abackerli, A.J., Shakarji, C., Sawyer, D. (June 25 -26, 2003). The Validation of CMM Task Specific Measurement Uncertainty Software. In Proc. of the ASPE 2003 summer topical meeting “Coordinate Measuring Machines", Charlotte, North Carolina.

[7] Ramu, P., Yagüe, J.A., Hocken, R.J., Miller, J. (2011). Development of a parametric model and virtual machine to estimate task specific measurement uncertainty for a five-axis multi-sensor coordinate measuring machine. Precis. Eng., 35(3), 431-439.

[8] Schwenke, H., Neuschaefer-Rube, U., Pfeifer, T., Kunzmann H. (2002). Optical Methods for Dimensional Metrology in Production Engineering. CIRP Ann. - Manuf. Technol., 51(2), 685-699.

[9] Wilhelm, R.G., Hocken, R., Schwenke, H. (2001). Task Specific Uncertainty in Coordinate Measurement. CIRP Ann. - Manuf. Technol., 50(2), 553-563.

[10] ISO/TS 15530- GPS - CMM. Techniques for determining the uncertainty of Measurement.

[11] ISO/IEC Guide 98-3:2008 - Uncertainty of measurement - Part 3: Guide to the Expression of Uncertainty in Measurement (GUM:1995).

[12] VDI/VDE 2617 Accuracy of coordinate measuring machines. Parameters and their reverification.

[13] Forbes, A. B., Hughes B., Sun, W. (2009). Comparison of measurements in co-ordinate metrology. Meas., 42(10), 1473-1477.

[14] Gąska A. (2009). Different uses of Monte Carlo Method in Coordinate Metrology. In Proc. of the X International Scientific Conference Automation in Production Planning and Manufacturing, Zilina.

[15] Sładek, J., Krawczyk, M., Ostrowska, K., Gąska, A. (2008). Usage of Monte Carlo Method in estimation of uncertainty of coordinate measurements in example of multiple measurement method. Scientific Books ATH Bielsko Biala, 84-93.

[16] Pressel, H.G., Hageney, T. (2008). Estimation of Uncertainty in Coordinate Measuring Technique. Expert Verlag.

[17] Hernla, M. (2007) Uncertainty of Coordinate Measurements. Expert Verlag.

[18] Hernla, M. (2000) Uncertainty estimation. QZ Qualitaet und Zuveraessigkeit, 45.

[19] Jakubiec, W. (2008). Analytical determination of measurement uncertainty in the coordinate measuring technique. Scientific Books 22 ATH Bielsko-Biala.

[20] Jakubiec W., Płowucha W., Starczak, M. (2012). Analytical estimation of coordinate measurement uncertainty. Measurement, 45, 2299-2308.

[21] Trapet, E., Franke, M., Hartig, F., Schwenke, H., Wadele, F., Cox, M., Forbers, A., Delbressine, F., Schnellkens, P., Trenk, M., Meyer, H., Morltz, G., Guth, Th., Wanner, N. (1999). Traceability of coordinate measuring machines according to the method of the Virtual Measuring Machines. PTB F-35, Braunschweig, Germany.

[22] Trenk, M., Franke, M., Schwenke, H. (2004). The "Virtual CMM", a Software Tool for Uncertainty Evaluation - Practical Application in an Accredited Calibration Lab. In Proc. of ASPE: Uncertainty Analysis in Measurement and Design.

[23] Sładek, J. (2011). The accuracy of coordinate measurements. Cracow University of Technology, Kraków, 495.

[24] Ostrowska, K. (2009). Assessment of measurement accuracy carried out using a coordinate measuring arms. Ph.D. dissertation, Cracow University of Technology.

[25] Muzyka-Żmudzki, M. (2008). The method of assessing the accuracy of large coordinate measuring machines using small standards. Ph.D. dissertation, Cracow University of Technology.

[26] Sładek, J., Krawczyk, M., Muzyka-Żmudzki, M., Gąska, A., Gacek, K., Kmita, A. (2009). The method of assessing the accuracy of large coordinate measuring machines using 2D standards. Journal of Machine Engineering, R14/2.

[27] Gąska, A. (2011). Modeling of accuracy of coordinate measurement with use of Monte Carlo Method. Ph.D. Dissertation. Cracow University of Technology.

[28] www.etalon-ag.com (March 2012) 
[29] Takatsuji, T., Goto, M., Kurosawa, T., Tanimura, Y. (1998). The first measurement of a three-dimensional coordinate by use of a laser tracking interferometer system based on trilateration. Measurement Science and Technology, 9(1), 38-41.

[30] Sładek, J., Gąska, A. (2012). Evaluation of coordinate measurement uncertainty with use of virtual machine model based on Monte Carlo method. Measurement, 45, 1564-1575.

[31] Sładek, J., Juras, B., Krawczyk, M., Gąska, A. (August 2010). Modeling of probing system errors by use of Monte Carlo Method. PAK. 